\title{
No receptor stands alone: IgG B-cell receptor intrinsic and extrinsic mechanisms contribute to antibody memory
}

\author{
Yinsheng $\mathrm{Xu}^{1,3, *}$, Liling $\mathrm{Xu}^{1,{ }^{*}}$, Meng Zhao ${ }^{1}$, ChenGuang $\mathrm{Xu}^{1}$, Yilin Fan ${ }^{1}$, Susan K Pierce ${ }^{2}$, Wanli Liu ${ }^{1,3}$ \\ ${ }^{1}$ MOE Key Laboratory of Protein Science, School of Life Sciences, Tsinghua University, Beijing 100084, China; ${ }^{2}$ Laboratory of Im- \\ munogenetics, National Institute of Allergy and Infectious Diseases, NIH, Rockville, MD 20852, USA; ${ }^{3}$ Collaborative Innovation \\ Center for Diagnosis and Treatment of Infectious Diseases, Tsinghua University, Beijing 100084, China
}

Acquired immunological memory is a striking phenomenon. A lethal epidemic sweeps through a naïve population, many die but those who survive are never "attacked twice - never at least fatally", as the historian Thucydides observed in 430 BCE. Antibody memory is critical for protection against many human infectious diseases and is the basis for nearly all current human vaccines. Antibody memory is encoded, in part, in isotype-switched immunoglobulin (Ig)G-expressing memory B cells that are generated in the primary response to antigen and give rise to rapid, high-affinity and high-titered antibody responses upon challenge with the same antigen. How IgG-B-cell receptors (BCRs) and antigen-induced IgG-BCR signaling contribute to memory antibody responses are not fully understood. In this review, we summarize exciting new advances that are revealing the cellular and molecular mechanisms at play in antibody memory and discuss how studies using different experimental approaches will help elucidate the complex phenomenon of B-cell memory.

Keywords: B-cell receptor; antibody response; B-cell memory; plasma cell; germinal center

Cell Research (2014) 24:651-664. doi:10.1038/cr.2014.65; published online 20 May 2014

\section{Introduction}

One fundamental function of the adaptive immune system is the production of antibodies. Upon recognition of foreign antigens, activation of mature naïve B cells is initiated, resulting in the production of short-lived plasma cells, the proliferation of naïve B cells and their differentiation into memory B cells and long-lived plasma cells [1] that constitute antibody $(\mathrm{Ab})$ memory responses [2]. B-cell memory is critical for protection against many human infectious diseases and is the basis for nearly all current human vaccines, suggesting the vital importance of biological studies on B-cell memory [2]. For instance, we do not yet have vaccines to prevent AIDS or malaria, both of which may depend on the ability to generate effective, broadly neutralizing memory $\mathrm{Ab}$ responses to

*These two authors contributed equally to this work.

Correspondence: Susan K Pierce ${ }^{\mathrm{a}}$, Wanli Liu ${ }^{\mathrm{b}}$

${ }^{a}$ Tel: 301-496-9589; Fax: 301-402-0259

E-mail: spierce@nih.gov

${ }^{\mathrm{b}}$ Tel: 86-10-6277-2730

E-mail: liuwanli@biomed.tsinghua.edu.cn the pathogens that cause these diseases. At present we still do not fully understand what properties of memory $\mathrm{B}$ cells underlie $\mathrm{Ab}$ memory characterized by the rapid, high-affinity and high-titered $\mathrm{Ab}$ responses. One distinguishing feature of many memory $\mathrm{B}$ cells is the expression of isotype-switched immunoglobulin (Ig)G B-cell receptors (BCRs). Therefore, the question of how B cells remember is often framed as: how do IgG-BCRs contribute to B-cell memory? This question has been extensively investigated using various tools of modern biological sciences and the results suggest that both intrinsic and extrinsic features of IgG-BCRs contribute to the efficient activation of memory $\mathrm{B}$ cells and their differentiation into plasma cells. In this review, we summarize these exciting findings. We first describe what is known about the differences in the structural features of IgM- and IgGBCRs and how these might be related to their functions. We then present a contemporary view of antigen-induced $\mathrm{B}$-cell signaling pathways and their similarities and differences in IgM-BCR- and IgG-BCR-expressing B cells. The impact of the form of the antigen that the $\mathrm{BCR}$ engages on signaling outcomes will also be addressed. Lastly we describe the intrinsic and extrinsic features of 
IgG-BCRs, which contribute to Ab memory responses. We believe that a clear and thorough understanding of the mechanisms underlying the activation of memory B cells would contribute to the design of still badly needed vaccines for infectious diseases including AIDS and malaria.

\section{Structural features of IgM- and IgG-BCRs}

B cells distinguish self-antigen versus non-self-antigen through their clonally distributed, surface-expressed BCRs [3, 4]. The BCR is composed of a membrane-bound form of immunoglobulin $(\mathrm{mIg})$ and a covalently-linked heterodimer of $\operatorname{Ig} \alpha$ and $\operatorname{Ig} \beta$ in a $1: 1$ ratio of $\mathrm{mIg}: \operatorname{Ig} \alpha / \operatorname{Ig} \beta$ heterodimer (Figure 1 ). The $\mathrm{mIg}$ and the $\operatorname{Ig} \alpha / \operatorname{Ig} \beta$ heterodimer are associated through non-covalent interactions $[5,6]$. Primary Ab responses are the results of the activation of mature naïve B cells, while memory or secondary $\mathrm{Ab}$ responses result from the activation of memory B cells $[2,7]$. The mIg subunits of BCRs expressed by mature naïve $\mathrm{B}$ cells and most memory $\mathrm{B}$ cells are different. Mature naïve B cells express mIgMand $\mathrm{mIgD}$-containing BCRs (Figure 1). During a primary $\mathrm{T}$-cell-dependent $\mathrm{Ab}$ response, the interaction of antigen-specific $\mathrm{B}$ cells with $\mathrm{T}$ cells and other accessory cells in lymphoid organs results in the formation of shortlived plasma cells and germinal centers (GCs). In GCs, mature naïve $B$ cells irreversibly isotype-switch their surface BCRs to predominately express mIgG-BCRs (Figure 1). These IgG-BCR-expressing GC B cells then undergo rounds of antigen-driven selection linked with somatic hypermutation [7-9], resulting in high-affinity IgG-BCR-expressing memory B cells and long-lived
A

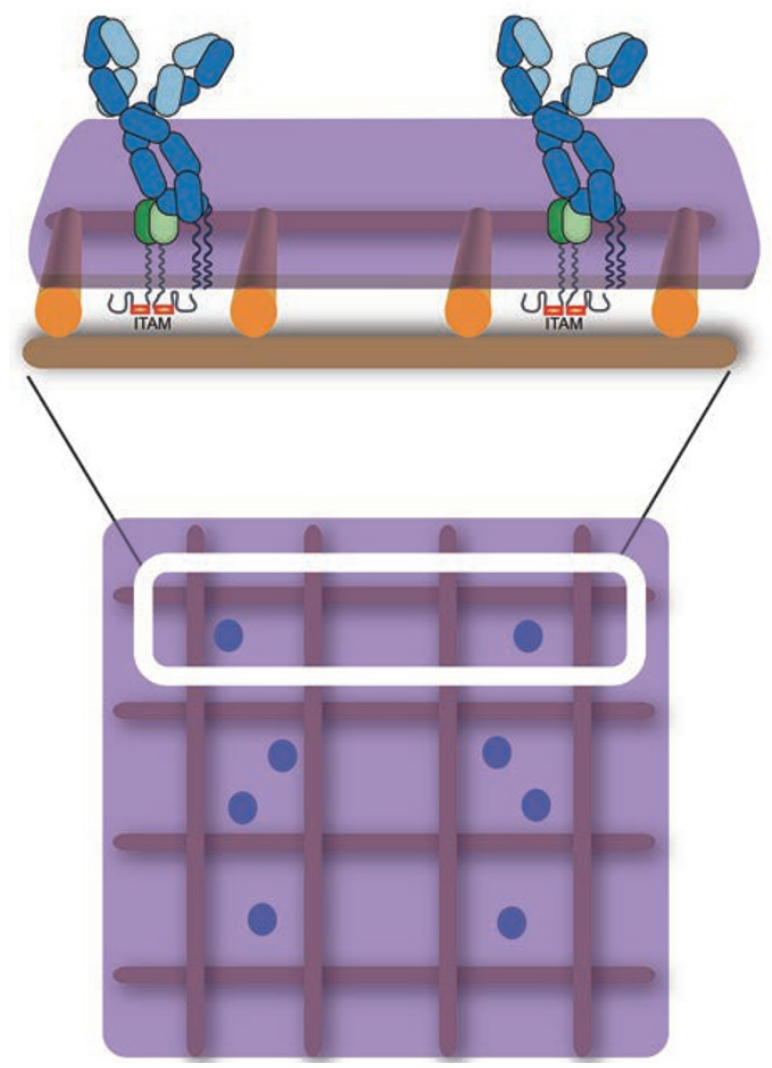

B

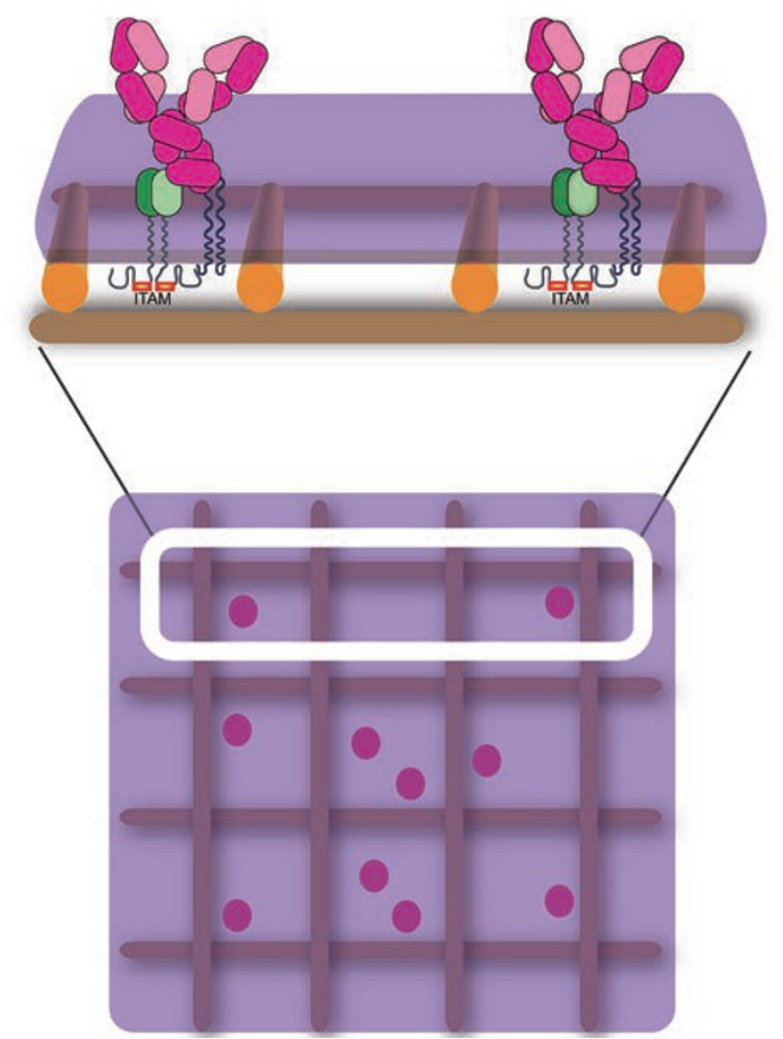

Figure 1 Schematic presentation of IgM-BCRs and IgG-BCRs. (A) IgM-BCRs composed of membrane-bound forms of IgM (light blue indicates the light chain, dark blue indicates the heavy chain) associated with a heterodimer of $\lg \alpha$ and $\lg \beta$ (green) on a mature naïve B cell. The mlg and the $\lg \alpha / \lg \beta$ heterodimer are associated through non-covalent interactions. (B) lgGBCRs composed of membrane-bound forms of lgG (light pink indicates the light chain, dark pink indicates the heavy chain) associated with the $\lg \alpha / \lg \beta$ heterodimer (green) on a memory B cell. In A and B, the BCRs are depicted in compartmentalized plasma membrane regions that are maintained by membrane-proximal actin fence and anchored transmembrane protein pickets as proposed by Kusumi et al. [79-81]. The ITAM-containing cytoplasmic tails of Ig $\alpha / \lg \beta$ heterodimer within the IgM- or IgG-BCR complexes are also shown. 
plasma cells.

Thus, there has been considerable focus on how the expression of IgG-BCRs by memory B cells contributes to $\mathrm{Ab}$ memory. Ultimately an understanding of how IgM- and IgG-BCRs function in antigen-driven B cell responses will require structural information of these receptors, which is currently unavailable. However, some features of IgG and IgM BCRs have been revealed to be implicated in distinguishing intrinsic functions of these receptors. Both IgM- and IgG-BCRs contain identical $\operatorname{Ig} \alpha / \operatorname{Ig} \beta$ heterodimers that link the BCR to the B-cell signaling apparatus through immunoreceptor tyrosine activation motifs (ITAMs) in $\operatorname{Ig} \alpha / \operatorname{Ig} \beta$ cytoplasmic domains [10]. However, mIgM and $\mathrm{mIgG}$ have different extracellular, transmembrane (TM) and cytoplasmic domains [7] (Figure 1). Both $\mathrm{mIgG}$ and $\mathrm{mIgM}$ have similar antigen-binding fragments (Fabs) consisting of $\mathrm{VH}$ and either $\mathrm{CH} \gamma 1$ or $\mathrm{CH} \mu 1$. The fragment crystallizable region ( $\mathrm{Fc}$ region) of $\mathrm{mIgG}$, composed of $\mathrm{CH} \gamma 2-3$, is shorter than that of mIgM, composed of $\mathrm{CH} \mu 2-4$ (Figure 1). However, our earlier studies showed that the Fc regions of $\mathrm{mIgM}$ and $\mathrm{mIgG}$ seem to be comparably efficient in triggering the early growth phase of BCR microclusters upon BCR-antigen engagement [11]. In $\mathrm{mIgG}$, the Fab and $\mathrm{Fc}$ are linked by a hinge region that is flexible and has no defined secondary structure. In contrast, in mIgM the flexible linker is replaced by a more rigid $\mathrm{C} \mu 2$ domain containing a conserved Asparagine (N)-linked glycosylation site that is absent in the flexible hinge region of $\mathrm{mIgG}$. It remains unknown how differences in the hinge region between $\mathrm{mIgM}$ and $\mathrm{mIgG}$ will affect their signal transduction capabilities, despite the efforts that have been placed to clarify this point [12].

The heavy chain of IgM is more heavily glycosylated than that of $\mathrm{IgG}$, with the heavy chain glycosylation accounting for $12 \%-14 \%$ of the molecular weight of IgM, as compared to only $2 \%-3 \%$ for IgG [12]. In soluble secreted antibodies, the glycans are multifunctional and play crucial roles in secreted $\mathrm{Ab}$ functions [12], but it is not known whether differences in glycosylation will affect the ability of IgM- and IgG-BCRs to initiate signaling. Our preliminary studies suggested that both mIgM- and mIgG-BCRs rely on the glycosylation of Ig molecules to efficiently initiate B-cell activation and interestingly mIgM-BCRs seem to be more dependent on glycosylation than $\mathrm{mIgG}-\mathrm{BCRs}$ in terms of the formation of signaling-active BCR microclusters and the maturation of B-cell immunological synapse.

Glycosylation also plays important roles in pre-BCR signaling. The mIgM molecule of pre-BCRs is composed of IgM heavy chains and a surrogate light chain. PreBCRs spontaneously initiate signaling and drive B-cell development, which depends on a conserved N-linked glycosylation site at position 46 (N46) in mIgM heavy chain, with N46 numbered from the N-terminus of the constant region of IgM [13]. However, N46-glycosylation is not required for BCR signaling in mature B cells, suggesting its specific requirement for pre-BCR function in developing B cells [13]. The surrogate light chain in pre-BCR accounts for the different requirements of N46-glycosylation in signaling mediated by pre-BCRs versus BCRs with the latter using a normal light chain. Indeed, there is evidence that the arginine-rich tail of the surrogate light chain $\lambda 5$ is also essential for pre-BCR signaling, suggesting the pivotal importance of both the $\lambda 5$ tail and the N46-glycosylation of mIgM heavy chain in pre-BCR signaling [13]. This same study also showed that the heavy chains of $\mathrm{mIgM}$ and $\mathrm{mIgD}$ are glycosylated differently and such differences are important for their respective functions [13].

The BCR's $\operatorname{Ig} \alpha / \operatorname{Ig} \beta$ heterodimer interacts with the membrane-proximal $\mathrm{CH}$ domains of $\mathrm{mIgM}$ and $\mathrm{mIgG}$ ( $\mathrm{CH} \mu 4$ and $\mathrm{CH} \gamma 3$, respectively) as well as with their TM regions (Figure 1). We recently provided evidence for extensive surface contacts between $\operatorname{Ig} \alpha / \operatorname{Ig} \beta$ and $\mathrm{mIg}$, involving multiple charged residues on both subunits of $\operatorname{Ig} \alpha / \operatorname{Ig} \beta$. In addition, we showed that the extracellular domains of $\operatorname{Ig} \alpha / \operatorname{Ig} \beta$ preferentially associate with $\operatorname{IgM}$ as compared to $\operatorname{IgG}$ [14]. At present it is not known whether these differences have functional consequences, either enhancing the activation of IgG-BCR signaling, or lowering the signaling threshold of the IgM-BCR and thus compensating for the lower affinity of IgM-BCRs to antigens during primary $\mathrm{Ab}$ responses.

Both mIgM and mIgD have three amino-acid cytoplasmic tails. In contrast, all mIgG subtypes have long cytoplasmic tails of 28 amino acids, which are highly conserved across species [15-18] (Figures 1 and 2). This remarkable conservation is stronger for the membrane-proximal region than the membrane-distal region (Figure 2). Indeed, the membrane-proximal region of the cytoplasmic tail of the evolutionarily diverged $\mathrm{mIgY}$ from lizard, Siamese crocodile and Chinese alligator is highly similar to that of mammalian mIgG. However, neither mIgA nor mIgE, both of which can be expressed as isotype-switched BCRs, shares sequence similarity with the cytoplasmic tail of $\mathrm{mIgG}$ (Figure 2). The question is: how does the mIgG tail contribute to memory $\mathrm{Ab}$ responses? The $\mathrm{mIgG}$ tail was shown to be required for the enhanced burst activity of IgG-BCR-expressing memory B cells in antigen recall memory Ab responses in mouse models $[19,20]$. In one study, two types of BCR transgenic ( $\mathrm{Tg}$ ) mice were made: wild-type (WT)mIgG1-BCR Tg mice that expressed mIgG1 with intact 


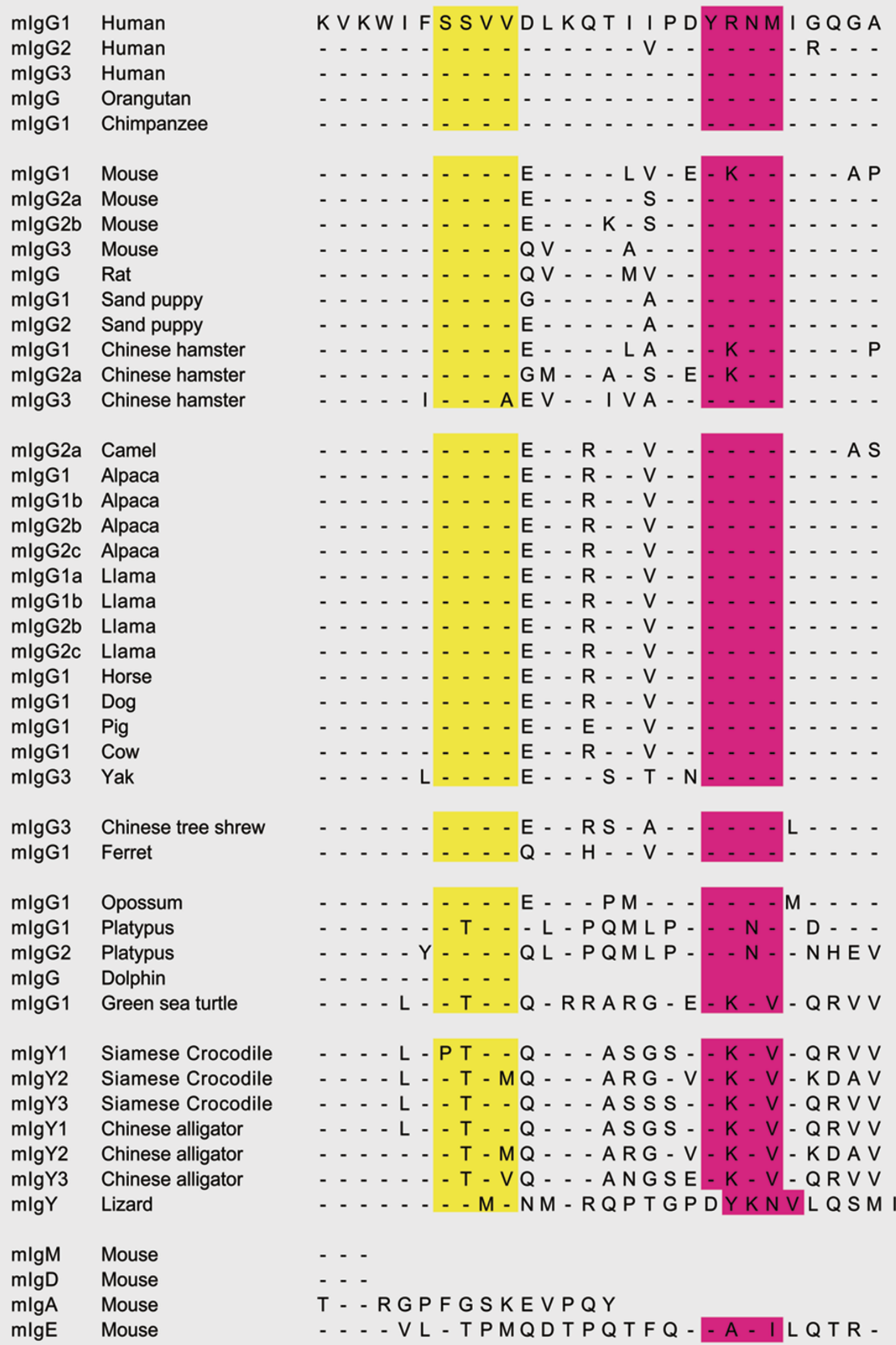

Figure 2 An alignment of the cytoplasmic tails of mlgs. Shown are alignments of the mlg tail sequences from different species that are available in NCBI. The SSVV motifs are highlighted in yellow and the ITT motifs in pink color. 
cytoplasmic tails and tailless-mIgG1-BCR Tg mice that expressed $\mathrm{mIgG1}$ with truncated cytoplasmic tails. When immunized with a T-cell-dependent antigen, chicken $\gamma$-globulin coupled to 4-hydroxy-3-nitro-phenylacetyl (NP), the tailless-mIgG1-BCR Tg mice showed significantly impaired NP-specific IgG1 secondary Ab responses compared to WT-mIgG1-BCR Tg mice [19]. In another study, three types of BCR Tg mice were made: WT-mIgM-BCR and WT-mIgG1-BCR Tg mice that expressed $\mathrm{mIgM}$ and $\mathrm{mIgG1}$ with the intact cytoplasmic tails, respectively, and IgM-IgG1-BCR Tg mice that expressed a chimeric mIg containing the ecto-domain of IgM and the TM domain and cytoplasmic tail of mIgG1. In adoptive transfer experiments, B cells from IgMIgG1-BCR Tg mice generated increased numbers of plasma blasts, which subsequently enhanced the magnitude of the memory $\mathrm{Ab}$ response compared to the $\mathrm{B}$ cells from WT-IgM-BCR Tg mice [20]. Moreover, another study [21] showed that the expression of mIgG1 with the intact cytoplasmic tail was able to partially replace the requirement for the $\operatorname{Ig} \alpha / \operatorname{Ig} \beta$ heterodimer during B-cell development, suggesting that the mIgG1 cytoplasmic tail independently interacts with cytoplasmic BCR signaling-related molecules. These studies demonstrate that the cytoplasmic tail of mIgG1 is both required and sufficient for an increased burst size in IgG-BCR-expressing B cells in response to antigens.

\section{A contemporary view of antigen-induced B-cell sig- naling pathways}

It is well-established that the binding of antigen to the BCR triggers signaling cascades that ultimately lead to B-cell proliferation and differentiation. Almost all of what we currently know about the key players of the BCR signaling pathways comes from biochemical studies of B cells activated by multivalent antigens in solution. These studies showed that antigen binding-induced BCR signaling is a multistep cascade, involving the participation of a series of protein tyrosine kinases (PTKs), serine-threonine kinases and lipid kinases, the recruitment of a number of adaptor proteins, which leads to the amplification and extension of BCR signaling, and finally the transcription of downstream target genes. As several published reviews have already elegantly summarized and discussed in detail the individual components in BCR signaling pathways [4, 22-26], here we will only briefly outline the basic features of BCR signaling pathways. In addition, we will focus on the new findings in the past few years to dissect the uniqueness of IgGBCR signaling in memory B cells. We will also discuss how these newly identified signaling molecules and tran- scription factors could be integrated into the overall BCR signaling cascades.

In brief, three different families of PTKs, Src, Syk, and Tec, are activated following BCR and antigen binding (Figure 3). The first kinases activated following BCR crosslinking are members of the Src family including Lyn, Blk and Fyn. Phosphorylation of ITAM in the cytoplasmic domains of $\operatorname{Ig} \alpha / \operatorname{Ig} \beta$ by activated $\operatorname{Lyn}$ generates phospho-tyrosine (pTyr) motifs that allow the binding of the SH2 domains of the second kinase, Syk, resulting in Syk's rapid auto-phosphorylation at multiple tyrosines within its linker regions [27]. Syk phosphorylation provides important $\mathrm{SH} 2$ domain-docking sites for the recruitment and activation of downstream signaling molecules, e.g., Bruton's tyrosine kinase (Btk) [28] and phospholipase $\mathrm{C} \gamma 2$ (PLC $\gamma 2$ ) [29] (Figure 3). PLC2 hydrolyzes phosphatidylinositol 4,5-biphosphate $\left(\mathrm{PIP}_{2}\right)$ to generate two second messengers, inositol 1,4,5-trisphosphate $\left(\mathrm{IP}_{3}\right)$ and diacylglycerol (DAG). $\mathrm{IP}_{3}$ binds to its receptor $\left(\mathrm{IP}_{3} \mathrm{R}\right)$ on the endoplasmic reticulum, triggering $\mathrm{Ca}^{2+}$ mobilization, which subsequently leads to the activation of NF-AT, partially through calmodulin and calcineurin. DAG recruits protein kinase $\mathrm{C}$ family members that subsequently activate CARMA1, Bcl10 and MALT1 (CBM) complexes, leading to the activation of $\mathrm{NF}-\kappa \mathrm{B}$ (Figure 3). The recruitment of a series of guanine nucleotide exchange factors (GEFs) including RasGRP by DAG and SOS by Grb2 to the membrane-proximal BCR signaling signalosome activates the small GTPase Ras, which subsequently leads to the activation of mitogen-activated protein (MAP) kinases. Btk is also a PTK that is activated and recruited to Syk membrane-proximal signalosomes to promote the phosphorylation of PLC2 (Figure 3). Starting from the Syk membrane-proximal signalosome, BCR signaling diversifies, resulting in the triggering of at least four different downstream signaling cascades $[22,23]$, toward the transcriptional activation or regulation of NF- $\mathrm{kB}, \mathrm{NF}-\mathrm{AT}$, FoxO (see below for details) and MAPK pathways (Figure 3).

Membrane-proximal BCR signaling also involves the activation of the $\mathrm{p} 85 \alpha / \mathrm{p} 110 \delta$ heterodimeric isoform of phosphatidylinositol (PI) 3 kinase (PI3K), which represents a key component of BCR signaling cascades. Although it is not completely clear how PI3K is recruited to the membrane-proximal BCR signalosome, it is evident that both CD19 and BCAP are required for the efficient activation of PI3K [30]. Activated PI3K converts $\mathrm{PIP}_{2}$ to $\mathrm{PIP}_{3}$, the latter serving as a membrane-anchored ligand for a subset of signaling proteins harboring the pleckstrin homology $(\mathrm{PH})$ domain. The $\mathrm{PH}$ domain-containing kinase Akt plays key roles in BCR signaling cascades by activating the mammalian target of rapamycin 


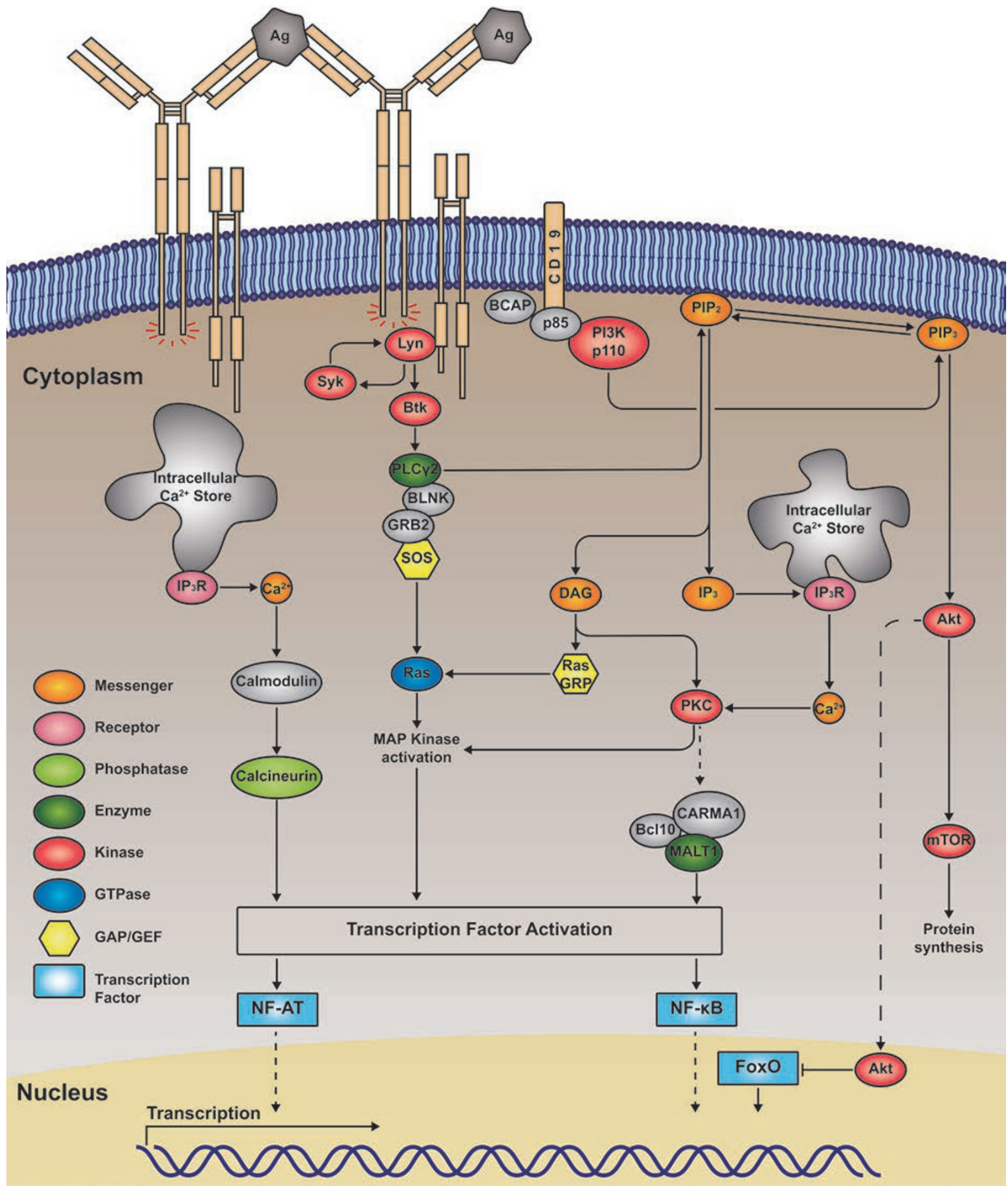

Figure 3 A contemporary view of antigen-binding-induced activation of BCR signaling pathways. A highly simplified version of the view of BCR signaling pathways is given. Early biochemical studies indicated that PTKs from three different families, including Lyn, Syk and Btk, are activated following BCR and antigen recognition, forming the typical membrane-proximal signalosome. Such signalosome complex is further stabilized by the involvement of a series of adaptor proteins including BLNK and GRB2. Upon the recruitment of PI3K and PLC $\gamma 2$ to these membrane-proximal signalosome complexes, these two signaling molecules are activated, the former leading to the production of $\mathrm{PIP}_{3}$ from $\mathrm{PIP}_{2}$, while the latter resulting in the production of $\mathrm{IP}_{3}$ and $\mathrm{DAG}$ from $\mathrm{PIP}_{2}$. Starting from this point, BCR signaling gets quite diverse and triggers at least four different downstream signaling cascades $[22,23]$, toward the transcriptional activation or regulation of NF- $\mathrm{B}$, NF-AT, FoxO and MAPK pathways. 
(mTOR) to regulate protein synthesis and subsequently cell proliferation. Another well-documented function of activated Akt is to phosphorylate and inactivate the nucleus-localized transcription factor FoxO. FoxO family members are known for their functions in maintaining the quiescence state of inactivated, resting B cells. Thus it is highly likely that the main function of the PI3K and Akt signaling cascade is to drive resting B cells into proliferation.

\section{The diverse nature of antigens that $B$ cells encoun- ter in vivo}

It is well-appreciated that the antigenic epitopes that B cells recognize are highly diverse in terms of the affinity $[16,31]$ for the BCR, the valency $[16,31]$ and density $[16,31]$ of the epitopes, the mechanical properties of the substrates [32] and whether the antigen is recognized in solution or on the surface of an antigen-presenting cell (APC) [33]. It is important to understand how these features of antigens affect the initiation of B-cell activation and to appreciate the diverse nature of antigens that $\mathrm{B}$ cells encounter in vivo.

Recent studies using advances in intravital imaging demonstrated that antigens enter lymph nodes by several routes and that B cells encounter antigens either in soluble form or on the surfaces of APCs depending on the nature of the antigen [34]. Small soluble antigens, like bacterial toxins, rapidly diffuse into lymph nodes through the afferent lymph vessel and thus encounter the B cells in soluble form [34-37]. However, large antigens, like viruses, bacteria and $A b$-antigen immune complexes, are only accessible to B cells within the lymph nodes through active presentation by macrophages and dendritic cells lining the subcapsular sinuses [38-41]. When these large antigens are captured and tethered to the surfaces of APCs, the biophysical motile properties of the antigens will be quite different from soluble antigens. Small soluble antigens are freely diffusing in three dimensions, in contrast to antigens on APC surfaces, which are restricted to lateral diffusion in two dimensions [33]. Soluble antigens that are unable to exert any substantial force on the BCRs allow free diffusion of BCRs. In contrast, antigens bound to APC surfaces restrict the diffusion of the BCRs and by doing so inevitably apply opposing forces to BCRs. We recently showed that activation of $\mathrm{B}$ cells is regulated by the mechanosensing capability of these cells [32], providing a mechanism by which BCR signaling can be affected by the context in which the antigen is recognized either in solution or on APC surfaces. A study from Batista and colleagues showed that B cells first spread over the surfaces of APCs bearing membrane-bound antigens and then contracted, which was not observed in B cells encountering soluble antigens [31]. In addition, B cells appear to use different mechanisms to enhance signaling when encountering soluble versus membrane-bound antigens. Our early study showed that the association of the BCR microclusters with lipid rafts required Src kinase activity when cells were stimulated by soluble antigens [42], but not by membrane-bound antigens [43]. Batista and colleagues showed that the BCR-activation co-receptor CD19 was required for B cell activation by membrane-bound antigen, but not by soluble antigen [44]. They found that BCR microclusters only signal after they colocalized with CD19 microclusters in B cells encountering membrane-bound antigens [44]. Using super-resolution microscopy, they further demonstrated that the local convergence of BCR and CD19 to Syk nanoclusters is an essential step in B-cell activation induced by membrane-bound antigens [45]. It is not completely clear why the initiation of BCR signaling has distinct requirements for B cells encountering soluble versus membrane-bound antigens, and what the consequence of their distinct requirements is. It is possible that the contribution of the membrane cytoskeleton network is more substantial for the quality and strength of BCR signaling in response to membrane-bound antigens as compared to soluble antigens. For example, pre-treatment of B cells with the actin polymerization inhibitor, latrunculin $B$, enhanced $\mathrm{BCR}$ signaling in response to soluble antigens, resulting in prolonged calcium increases and ERK activation [46]. In contrast, latrunculin B treatment significantly impaired B-cell responses to membrane-bound antigens $[16,31]$. The intense involvement and requirement of the membrane cytoskeleton network in BCR signaling activation will likely modify many unidentified features of the BCR signaling cascades, an area that deserves further investigation. Similarly, responses to membrane-bound antigens, but not to soluble antigens, likely involve mechanosensing, for example through the integrin system that will also change the quality of BCR signaling. CD19 is a key BCR-activation co-receptor and plays essential roles in activating the PI3K-Akt signaling pathway. The essential requirement and involvement of CD19 in BCR signaling induced by membrane-bound antigens, but not by soluble antigens, indicates different features of BCR signaling in these two cases.

The valency of antigens also plays key roles in affecting the quality of BCR signaling. Although it has been widely observed that in solution multivalent antigens are more potent B-cell activators than monovalent antigens, it is only recently that the underlying mechanism has been revealed. It was shown that multivalent antigens ef- 
ficiently cluster the BCRs and subsequently the clustered BCRs initiate a positive feedback to boost Syk activation, thus extricating the dependence on Src family kinases within the membrane-proximal BCR signalosomes. In contrast, monovalent antigens fail to induce BCR clustering, leading to more substantial requirements of both Syk and Src family kinases [47].

\section{The nature of IgG- versus IgM-BCR-induced sig- naling}

Several studies have compared the signaling cascades of IgM-BCR-expressing mature naïve B cells and IgG-BCR-expressing memory B cells upon soluble multivalent antigen binding in biochemical experiments using either B-cell lines or primary B cells in vitro. Three independent studies provided evidence for differential BCR signaling in IgM-BCR- and IgG-BCR-expressing B cells, which is due to the presence of the conserved cytoplasmic tail in $\mathrm{mIgG}[21,48,49]$. One early study using the B-cell lymphoma cell line, K46, reported a mechanism by which the $\mathrm{mIgG}$ cytoplasmic tail dramatically enhances BCR signaling by preventing CD22-mediated inhibition [48]. This study suggests that the crosslinking of IgM-BCRs but not IgG-BCRs induces the phosphorylation of the immunoreceptor tyrosine-based inhibition motif (ITIM) in the cytoplasmic domain of CD22, resulting in the recruitment of protein phosphatases, including Src homology phosphatase-1 (SHP-1), and the subsequent downregulation of ERK activation and intra-
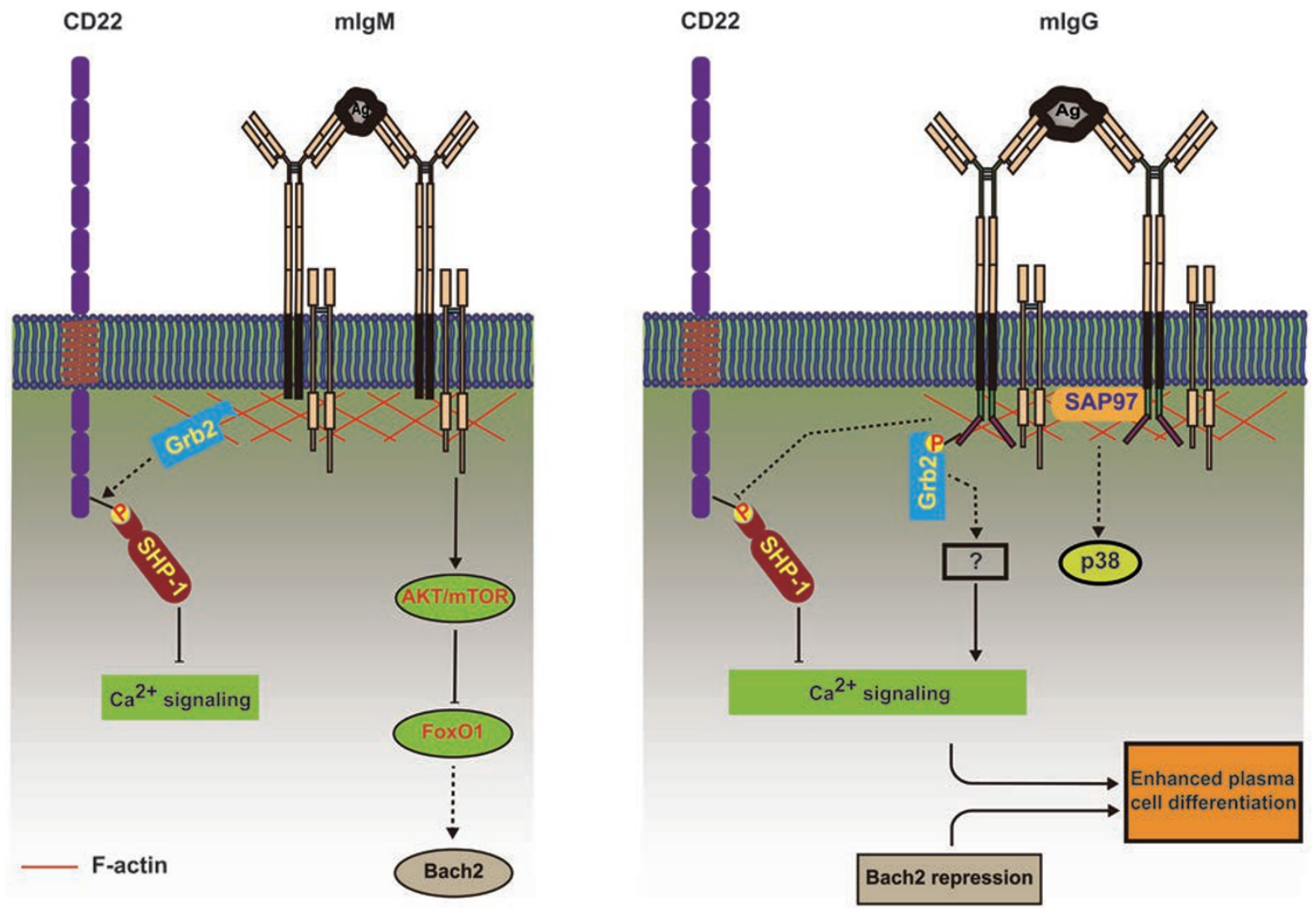

Figure $4 \mathrm{~A}$ simplified summary of IgG-BCR-intrinsic and -extrinsic factors that work together to enhance the activation and differentiation of memory B cells. Most of the IgG-BCR-intrinsic and -extrinsic factors discussed in this review are depicted in this cartoon to show their potential location and interacting proteins in enhancing the activation and differentiation of IgG-BCR-expressing memory B cells, but not IgM-BCR-expressing mature naïve B cells. IgG-BCR-intrinsic factors likely involve the adaptor protein Grb2 recruited through the membrane distal ITT motif and the scaffolding protein SAP97 recruited through the membrane-proximal SSVV motif of mlgG cytoplasmic tail. These IgG-BCR-intrinsic factors may be responsible for the enhanced calcium responses through some unidentified mechanisms. IgG-BCR-extrinsic factors likely involve the transcription factor Bach2, the expression of which is repressed in IgG-BCR-expressing memory B cells but not in lgM-BCR-expressing mature naïve B cells. The repression of Bach2 in memory B cells is required for enhanced plasma cell differentiation of these cells. 
cellular $\mathrm{Ca}^{2+}$ mobilization. The failure of IgG-BCRs to activate $\mathrm{CD} 22$ leads to enhanced $\mathrm{B}$-cell activation (Figure $4)$. These observations were also confirmed in WEHI279, BAL17, A20 cell lines and mouse spleen primary B cells. Two later studies using B cells from Ig Tg mice with IgM-BCRs or chimeric IgMG-BCRs with or without $\mathrm{CD} 22$ deficiency showed that the $\mathrm{mIgG}$ tail enhanced intracellular calcium responses upon BCR crosslinking independent of CD22 [21, 49]. A possible explanation for these inconsistent findings is that the loss of CD22 might be functionally compensated during B-cell development in CD22-deficient mice, but not in cell lines in which the expression of CD22 is knocked down. Studies in mouse primary B cells showed that calcium responses are significantly enhanced in antigen-stimulated IgG-BCR-expressing memory B cells compared to IgM-BCR-expressing mature naïve $B$ cells, although no differences in ERK phosphorylation were observed. Thus, despite the inconsistency regarding the function of CD22 in the enhanced activation of memory B cells, it appears clear that IgG-BCR-expressing memory B cells show significantly enhanced calcium responses upon multivalent soluble antigen binding (Figure 4), and this enhanced effect depends on the conserved cytoplasmic tail of $\mathrm{mIgG}[21,48,49]$. By microarray-based transcription profiling, Goodnow and colleagues found that the transcription of as many as $\sim 50 \%$ of the antigen-induced

Table 1 Genes that are strongly induced in IgM-BCR-expressing $\mathrm{B}$ cells but not in mIgM-mIgG B cells upon BCR and antigen recognition

\begin{tabular}{ll}
\hline Gene name & \multicolumn{1}{c}{ Annotation } \\
\hline Irf4 & Interferon regulatory factor \\
uPAR & Urokinase receptor \\
Mafk & Transcription factor \\
Nur77 & Nuclear receptor subfamily 4 group A member 1 \\
Gfi1 & Transcription repressor \\
Gadd153 & Growth arrest and DNA damage induced gene \\
Myd116 & Growth arrest and DNA damage induced gene \\
Snk & Polo-like kinase \\
Tis7 & Tetradecanoyl phorbol acetate-induced sequence \\
Ccl3 & Chemokine (C-C motif) ligand \\
Ccl4 & Chemokine (C-C motif) ligand \\
Ccr7 & Chemokine receptor \\
Ccr9 & Chemokine receptor \\
Cd83 & Cell surface lectin \\
I BB 3 & NF- BB signaling regulator \\
Atf3 & Cyclic AMP-dependent transcription factor \\
Atf4 & Cyclic AMP-dependent transcription factor \\
\hline
\end{tabular}

genes in IgM-BCR-expressing B cells was not increased in cells which expressed engineered $\mathrm{mIgM}$ containing the cytoplasmic domain of mIgG1 [49] (Table 1). Taken together, these studies suggest that the cytoplasmic tail of $\mathrm{mIgG}$ exerts a dual-role in the activation of B cells by enhancing the transcription of some B cell genes while suppressing others $[21,49]$.

The mechanism by which the cytoplasmic tail of $\mathrm{mIgG}$ affects the outcome of antigen binding is of substantial interest. As mentioned above, earlier studies provided evidence that the $\mathrm{mIgG}$ tail is required for antigen recall memory $\mathrm{Ab}$ responses $[19,20]$. However, only recently mechanistic explanations for this requirement were provided. The first evidence for a direct interaction of the $\mathrm{mIgG}$ cytoplasmic tail with a component of the BCR signaling cascade came from the study by Wienands and his colleagues [50], which showed that a conserved Ig tail tyrosine (ITT) motif in the cytoplasmic domain of both $\mathrm{mIgG}$ and $\mathrm{mIgE}$ (Figure 2) was phosphorylated upon IgG- or IgE-BCR crosslinking by multivalent soluble antigens. The phosphorylated ITT motif recruits the adaptor protein Grb2, leading to the enhancement of calcium responses and B-cell proliferation (Figure 4).

Using total internal reflection fluorescence-based high-resolution high-speed live-cell imaging, we recently showed that the earliest events that occur following antigen binding to the BCR are highly sensitive to BCR isotypes [16]. By single-particle tracking, we found that, as compared to IgM-BCRs, IgG-BCRs showed a dramatically enhanced ability to oligomerize and the resulting IgG-BCR microclusters grew more rapidly, ultimately resulting in an increased recruitment of Syk to the immunological synapse and more robust calcium responses [11]. Through extensive domain-swapping and mutagenesis analyses, we found that 15 membrane-proximal residues of the $\mathrm{mIgG}$ tail conferred enhanced oligomerization [11]. This 15-residue region does not contain the ITT motif that is phosphorylated following IgG-BCR crosslinking [50]. Alignment of the known sequences of the cytoplasmic domains of $\mathrm{mIgG}$ from different species shows a remarkably conserved SSVV motif at the membrane-proximal region of the cytoplasmic tail of mIgG. SSVV seems to be a partial match to type I postsynaptic density 95/disc large (Dlg)/zona occludens 1 (PDZ) domain-binding motif, X-S/T-X-V [51, 52] (Figure 2). However it shall be noted that SSVV is not a canonical type I PDZ domain-binding motif as it does not contain a C-terminal $\mathrm{COOH}$ group, and thus a binding mechanism of the SSVV motif deserves further explorations. In contrast, the tail of mIgE is different in this region although it contains the ITT motif (Figure 2). Thus, our studies revealed a previously unappreciated function of 
the membrane-proximal region of the $\mathrm{mIgG}$ tail in $\mathrm{IgG}$ BCR signaling (Figure 2).

We thus speculated that the membrane-proximal region of the mIgG tail binds to a PDZ domain-containing protein. Through a series of bioinformatics, mass spectroscopy, phage display screening and fluorescence lifetime imaging microscopy (FLIM)-based fluorescence resonance energy transfer (FRET) analyses, we identified a PDZ domain-containing protein synapse-associated protein 97 (SAP97, also known as DLG1 or DLGH1), a member of the conserved membrane-associated, guanylate-kinase (MAGUK) family, as a potential binding partner of the mIgG tail [15] (Figure 4). Little was known about the function of SAP97 in B cells. In neurons, SAP97 has been shown to bind to the cytoplasmic tails of neuronal N-Methyl-D-aspartic acid (NMDA) and $\alpha$-amino-3-hydroxy-5-methyl-4-isoxazolepropionic acid (AMPA) receptors, and thus plays key roles in controlling receptor density and signal strength at neuronal synapses [53].

We showed that IgG-BCR immune synapse recruited more SAP97 as compared to IgM-BCR immune synapse [15]. More importantly, SAP97 knockdown greatly diminished the ability of IgG-BCRs to oligomerize, the growth of the resulting clusters, and the recruitment of kinases, but had no effect on the function of IgM-BCRs. Intriguingly, studies of the kinetics of antigen-driven BCR cluster growth in WT and SAP97-deficient IgG-BCR-expressing B cells showed that SAP97's function was not required in early BCR signaling when oligomerization and initial clustering occurred (before 5 min), but became essential for efficient clustering subsequently (unpublished observations from $\mathrm{Xu}$ and $\mathrm{Liu}$ ). These results lead us to propose that SAP97 serves as a scaffolding protein to stabilize the antigen-driven formation of IgG-BCR oligomers and to facilitate the assembly of the BCR signalosome. It will be interesting to further determine how SAP97 functions in IgG-BCR signaling.

\section{Memory Ab responses of Grb2 and SAP97 knock- out (KO) mice}

Taken together, the above evidence indicates that Grb2 and SAP97 enhance the activation of IgG-BCR-expressing memory B cells, with Grb2 being recruited to the conserved phosphorylated ITT motif in the membrane-distal region of $\mathrm{mIgG}$ and SAP97 being recruited to the conserved SSVV motif in the membrane-proximal region of $\mathrm{mIgG}$ (Figure 4). What effects do B cell-specific deficiencies of Grb2 and SAP97 have on memory $\mathrm{Ab}$ responses? For Grb2, Jang IK et al. [54] produced B-cell lineage-specific Grb2 KO mice by crossing Grb2- floxed mice to CD19-Cre mice (Grb2-CD19 KO mice), while Ackermann et al. generated B-cell lineage-specific Grb2 KO mice by crossing Grb2-floxed mice to mb1-Cre mice (Grb2-mb1 KO mice) [55]. Both types of B-cell lineage-specific Grb2 KO mice showed normal development of Pro-, Pre-, immature, marginal, and B1 B cells, with significantly reduced peripheral $\mathrm{T} 1, \mathrm{~T} 2$, and mature B cells. Both immature and mature B cells from Grb2CD19 KO mice showed enhanced proliferation upon anti-IgM or lipopolysaccharide (LPS) stimulation, while only immature but not mature B cells from Grb2-mb1 $\mathrm{KO}$ mice showed such enhanced proliferation. B cells from both lines showed enhanced calcium responses and reduced phosphorylation of CD22. Moreover, Grb2CD19 KO B cells showed significantly enhanced phosphorylation of PLC $\gamma 2$, ERK, c-Jun N-terminal protein kinase (JNK) and p38 signaling molecules, while Grb2mb1 KO B cells showed normal phosphorylation of ERK and $\mathrm{p} 38$, and attenuated phosphorylation of JNK. These biochemical analyses suggest that Grb2 most likely attenuates rather than enhances IgM-BCR signaling activation, consistent with the reported function of Grb2 in the literature [56, 57].

After immunization with T-dependent antigens, both types of Grb2 B cell KO mice showed impaired GC formation and thus fewer IgG-producing cells in the spleen. Both types of mice showed normal primary $\mathrm{Ab}$ responses. However, Grb2-CD19 KO mice showed normal secondary IgG responses and normal generation of high-affinity antibodies, while Grb2-mb1 KO mice showed impaired secondary IgG responses. These inconsistencies in memory responses but not in primary $\mathrm{Ab}$ responses may be caused by the different types of immunogens used in immunization experiments. In the experiments with Grb2-CD19 KO mice, model hapten antigen NP30-KLH pre-precipitated in standard adjuvant alum was injected into mice intraperitoneally in primary immunization and NP30-KLH in PBS without adjuvant was used in antigen recall immunization. In the experiments with Grb2mb1 KO mice, HCMV strain AD169-based virus-like particle (VLP) in PBS was injected intravenously in both primary and antigen recall immunization. As the form of antigens may significantly affect the requirements for the activation of $\mathrm{B}$ cells and the subsequent $\mathrm{Ab}$ responses as discussed above, it is perhaps not surprising to see these inconsistencies. Taken together, these two studies suggest that complicated and sophisticated molecular mechanisms are involved in the generation of memory $\mathrm{Ab}$ responses.

It will also be of substantial interest to determine the role of SAP97 in memory $\mathrm{Ab}$ responses in vivo. Based on our results in vitro, showing that SAP97 is required 
for the enhanced responses of IgG-BCR-expressing B cells, we hypothesize that SAP97-deficient B cells may not be able to survive $\mathrm{GC}$ reactions or perhaps more likely that SAP97-deficient B cells would not be able to compete with WT B cells in GC reactions. A recent study by Hawkins et al. [58] investigated SAP97-deficient (SAP97/Dlg1 KO) fetal liver chimera mice in which both $\mathrm{B}$ and $\mathrm{T}$ cells were reconstituted. B cells proliferated normally in vitro in response to LPS, or anti-CD40 and IL-4. Moreover, these mice showed no significant deficiencies in primary or secondary $\mathrm{Ab}$ responses, although these SAP97-deficient mice tended to produce less high-affinity Abs in secondary responses. These findings suggest that SAP97-deficiency does not block B cells from proceeding through GC reactions, and instead SAP97-deficient B cells likely would fail to compete with WT B cells in GC reactions. Indeed, in mice that carry targeted $\mathrm{Ab}$ genes with low or high antigen-binding affinity to NP hapten antigen, low- and high-affinity $B$ cells have the same intrinsic capacity to respond to antigen, but only high-affinity B cells accumulated in GCs when limited numbers of low- and high-affinity B cells were co-transferred into WT recipient mice [59]. Thus, we predict that in chimeras with both WT and SAP97-deficient B cells, only WT B cells would produce high-affinity memory $\mathrm{B}$-cell responses.

\section{The role of IgG-BCR extrinsic effects in memory Ab responses}

Although it appears clear that intrinsic features of the IgG-BCR contribute to Ab memory responses, it is likely that other features of memory B cells will also contribute to $\mathrm{Ab}$ memory. This issue was recently addressed by Kurosaki and colleagues who convincingly demonstrated that the pre-antigen experience-induced repression of the Bach2 transcription factor contributes to the heightened differentiation activity of IgG1 memory B cells [60]. In their studies, the authors used an elegant mouse model system of C1-Cre micexinducible diphtheria toxin receptor (iDTR) mice to specifically deplete the IgG1-BCR-expressing B cells. As expected, these mice were unable to mount antigen recall IgG1 $\mathrm{Ab}$ responses. Since antigen-experienced IgM-BCR-expressing $\mathrm{B}$ cells are intact in these mice, the authors concluded that IgG1-BCR-expressing memory B cells are the major source of the memory $A b$ responses [60]. Using an adoptive-transfer mouse model, they observed that IgG1-BCR-expressing memory B cells showed a higher propensity to differentiate into plasma cells compared to IgM-BCR-expressing mature naïve B cells, consistent with the observation from earlier studies [61-63]. The au- thors then asked: what is the behavior of IgG1-BCR-expressing $\mathrm{B}$ cells that have never encountered cognate antigens? The authors created IgG1-BCR embryonic stem cells (ESCs) by nuclear transfer from endogenous NP-specific IgG1-BCR-expressing B cells derived from C57BL/6 mice, and used one such ESC line to generate chimeric mice. These chimeric mice contained NP-specific IgG-BCR-expressing B cells that have never encountered the cognate antigen (termed IgG-BCR-ESC $B$ cells) [60]. By adoptive transfer experiments, they showed that NP-specific IgM-BCR-expressing B cells and IgG-BCR-ESC B cells undergo predominantly GC reactions rather than differentiation into plasma cells, suggesting that the expression of IgG1-BCR on the B cell surface alone probably cannot account for the heightened capacity of memory B cells to differentiate into plasma cells. Indeed, this speculation was further supported by the observation that the antigen-experienced IgG-BCRESC B cells differentiated more readily into plasma cells compared to antigen-inexperienced IgG-BCR-ESC B cells [60].

The differentiation of B cells into plasma cells is under the control of transcription factors with opposing effects. It is known that the expression of Blimp-1, IRF4 and XBP-1 is upregulated and required for plasma cell differentiation [64-66], while the expression of other transcription factors including Pax5, Bach2 and Bcl-6 is suppressed in plasma cells [67-69]. In an earlier study, Luckey et al. [70] examined both the up and downregulated transcripts of memory B cells compared to naïve, GC B cells and plasma cells. Their study suggests that the changes in gene expression profiles are surprisingly shared between memory B cells, memory $\mathrm{T}$ cells and long-term hematopoietic stem cells, suggesting a common molecular mechanism of self-renewal in all cases. Similarly, Bhattacharya et al. [71] examined the transcription profiles of mouse naïve, GC, memory B cells and plasma cells. They showed increased expression of AID, chemotactic receptors, co-stimulatory molecules and several anti-apoptotic genes in memory B cells. A recent study by Kurosaki and colleagues showed that reduction of Bach2 in IgG1 memory B cells promotes their differentiation into plasma cells [60] (Figure 4). Bach2 might not be the only IgG-BCR-extrinsic transcription factor that works to enhance memory immune responses. For example, expression of either Krüppel-like factor (Klf) 2 or Ski, two transcriptional regulators specifically enriched in memory B cells relative to their GC precursors, imparts a competitive advantage to BCR- and CD40-engaged B cells in vitro [71]. Tomayko et al. [72] suggested that memory B cells have increased expression of genes important for the regulation of adenosine signal- 
ing and cAMP responses. Furthermore, memory B cells upregulate receptors that are essential for ESC self-renewal [72].

The differences in gene expression profiles between human naïve and memory B cells have also been characterized [73-78]. For example, Tangye and Good [78] reported that compared to naïve B cells, memory B cells that were isolated from human spleen expressed lower levels of Klf4, Klf9 and promyelocytic leukemia zinc finger (PLZF), and other transcription factors that are important in maintaining cellular quiescence. Interestingly, all these genes are those that would be downregulated after B-cell activation through CD40 and the BCR. Enforced expression of Klf4, Klf9 and PLZF in memory B cells delayed their entry into cell division and reduced the number of proliferating cells [78].

\section{Summary and future perspectives}

Here we summarized the evidence that IgG-BCR-expressing memory $\mathrm{B}$ cells show enhanced immune activation upon antigen re-encounter and that the enhanced differentiation toward plasma cells is due to both IgG-BCR-intrinsic and -extrinsic features. BCR-intrinsic mechanisms likely involve both the adaptor protein Grb2 and the scaffolding protein SAP97, which function through the membrane-distal ITT motif and the membrane-proximal SSVV motif in the $\mathrm{mIgG}$ cytoplasmic tail, respectively. Memory B-cell-intrinsic, but IgG-BCR-extrinsic, mechanisms involve transcription factors such as Bach2. It is likely that other yet unidentified factors also contribute to the enhanced activation of memory B lymphocytes. Such factors could be IgG-BCR-intrinsic or -extrinsic or even extrinsic to the memory B cell itself, but at some degree relying on the microenvironment. The identification of these intrinsic and extrinsic features in regulating the activation and differentiation of IgG-BCR-expressing memory B cells will aid in our understanding of the intriguing nature of acquired immunological memory. Such information will also hopefully benefit efforts in developing vaccines to prevent the pandemics of AIDS and malaria.

\section{Acknowledgments}

This work was supported by the Ministry of Science and Technology of China (2014CB542500-03, 2014AA020527), the National Natural Science Foundation of China (81361120384, 31270913), the Beijing Natural Science Foundation (5132016), the $\mathrm{PhD}$ Programs Foundation of Ministry of Education of China (20130002110059, 201200021200076), the New Century Excellent Talents program in University of Ministry of Education of China (NCET-12-0299), the One-Thousand-Youth-Talents pro- gram (2069999-3) of Chinese Central Government, and Tsinghua University Initiative Scientifc Research Program (2013Z1089279). Wanli Liu expresses his heartfelt thanks to Dr Susan K Pierce for her inculcated mentoring in his postdoctoral trainings.

\section{References}

1 Pierce SK, Liu W. The tipping points in the initiation of B cell signalling: how small changes make big differences. Nat Rev Immunol 2010; 10:767-777.

2 McHeyzer-Williams LJ, McHeyzer-Williams MG. Antigen-specific memory B cell development. Annu Rev Immunol 2005; 23:487-513.

3 Reth M, Wienands J. Initiation and processing of signals from the B cell antigen receptor. Annu Rev Immunol 1997; 15:453479.

4 Cambier JC, Pleiman CM, Clark MR. Signal transduction by the B cell antigen receptor and its coreceptors. Annu Rev Immunol 1994; 12:457-486.

5 Schamel WW, Reth M. Monomeric and oligomeric complexes of the B cell antigen receptor. Immunity 2000; 13:5-14.

6 Tolar P, Sohn HW, Pierce SK. The initiation of antigen-induced B cell antigen receptor signaling viewed in living cells by fluorescence resonance energy transfer. Nat Immunol 2005; 6:1168-1176.

7 Kurosaki T, Aiba Y, Kometani K, et al. Unique properties of memory B cells of different isotypes. Immunol Rev 2010; 237:104-116.

8 Victora GD, Nussenzweig MC. Germinal centers. Annu Rev Immunol 2012; 30:429-457.

9 MacLennan IC. Germinal centers. Annu Rev Immunol 1994; 12:117-139.

10 Reth M. Antigen receptors on B lymphocytes. Annu Rev Immunol 1992; 10:97-121.

11 Liu W, Meckel T, Tolar P, et al. Intrinsic properties of immunoglobulin $\mathrm{IgG1}$ isotype-switched B cell receptors promote microclustering and the initiation of signaling. Immunity 2010; 32:778-789.

12 Arnold JN, Wormald MR, Sim RB, et al. The impact of glycosylation on the biological function and structure of human immunoglobulins. Annu Rev Immunol 2007; 25:21-50.

13 Ubelhart R, Bach MP, Eschbach C, et al. N-linked glycosylation selectively regulates autonomous precursor BCR function. Nat Immunol 2010; 11:759-765.

14 Radaev S, Zou Z, Tolar P, et al. Structural and functional studies of Igalphabeta and its assembly with the B cell antigen receptor. Structure 2010; 18:934-943.

15 Liu W, Chen E, Zhao XW, et al. The scaffolding protein synapse-associated protein 97 is required for enhanced signaling through isotype-switched IgG memory B cell receptors. Sci Signal 2012; 5:ra54.

16 Liu W, Meckel T, Tolar P, et al. Antigen affinity discrimination is an intrinsic function of the B cell receptor. J Exp Med 2010; 207:1095-1111.

17 Reth M. Antigen receptor tail clue. Nature 1989; 338:383.

18 Tarlinton D. Antigen presentation by memory B cells: the sting is in the tail. Science 1997; 276:374-375.

19 Kaisho T, Schwenk F, Rajewsky K. The roles of gamma 1 heavy chain membrane expression and cytoplasmic tail in 
IgG1 responses. Science 1997; 276:412-415.

20 Martin SW, Goodnow CC. Burst-enhancing role of the IgG membrane tail as a molecular determinant of memory. Nat Immunol 2002; 3:182-188.

21 Waisman A, Kraus M, Seagal J, et al. IgG1 B cell receptor signaling is inhibited by CD22 and promotes the development of B cells whose survival is less dependent on Ig alpha/beta. $J$ Exp Med 2007; 204:747-758.

22 DeFranco AL. The complexity of signaling pathways activated by the BCR. Curr Opin Immunol 1997; 9:296-308.

23 Dal Porto JM, Gauld SB, Merrell KT, et al. B cell antigen receptor signaling 101. Mol Immunol 2004; 41:599-613.

24 Kurosaki T, Shinohara H, Baba Y. B cell signaling and fate decision. Annu Rev Immunol 2010; 28:21-55.

25 Kurosaki T. B-lymphocyte biology. Immunol Rev 2010; 237:59.

26 Jumaa H, Hendriks RW, Reth M. B cell signaling and tumorigenesis. Annu Rev Immunol 2005; 23:415-445.

27 Saouaf SJ, Mahajan S, Rowley RB, et al. Temporal differences in the activation of three classes of non-transmembrane protein tyrosine kinases following B-cell antigen receptor surface engagement. Proc Natl Acad Sci USA 1994; 91:9524-9528.

28 Smith CI, Baskin B, Humire-Greiff P, et al. Expression of Bruton's agammaglobulinemia tyrosine kinase gene, BTK, is selectively down-regulated in T lymphocytes and plasma cells. J Immunol 1994; 152:557-565.

29 Weber M, Treanor B, Depoil D, et al. Phospholipase C-gamma2 and Vav cooperate within signaling microclusters to propagate B cell spreading in response to membrane-bound antigen. J Exp Med 2008; 205:853-868.

30 Castello A, Gaya M, Tucholski J, et al. Nck-mediated recruitment of BCAP to the BCR regulates the PI(3)K-Akt pathway in B cells. Nat Immunol 2013; 14:966-975.

31 Fleire SJ, Goldman JP, Carrasco YR, et al. B cell ligand discrimination through a spreading and contraction response. Science 2006; 312:738-741.

32 Wan Z, Zhang S, Fan Y, et al. B cell activation is regulated by the stiffness properties of the substrate presenting the antigens. $J$ Immunol 2013; 190:4661-4675.

33 Wan Z, Liu W. The growth of B cell receptor microcluster is a universal response of $\mathrm{B}$ cells encountering antigens with different motion features. Protein Cell 2012; 3:545-558.

34 Batista FD, Harwood NE. The who, how and where of antigen presentation to B cells. Nat Rev Immunol 2009; 9:15-27.

35 Clark SL Jr. The reticulum of lymph nodes in mice studied with the electron microscope. Am J Anat 1962; 110:217-257.

36 Farr AG, Cho Y, De Bruyn PP. The structure of the sinus wall of the lymph node relative to its endocytic properties and transmural cell passage. Am J Anat 1980; 157:265-284.

37 van Ewijk W, Brekelmans PJ, Jacobs R, et al. Lymphoid microenvironments in the thymus and lymph node. Scanning Microsc 1988; 2:2129-2140.

38 Carrasco YR, Batista FD. B cells acquire particulate antigen in a macrophage-rich area at the boundary between the follicle and the subcapsular sinus of the lymph node. Immunity 2007; 27:160-171.

39 Junt T, Moseman EA, Iannacone M, et al. Subcapsular sinus macrophages in lymph nodes clear lymph-borne viruses and present them to antiviral B cells. Nature 2007; 450:110-114.
40 Phan TG, Grigorova I, Okada T, et al. Subcapsular encounter and complement-dependent transport of immune complexes by lymph node B cells. Nat Immunol 2007; 8:992-1000.

41 Qi H, Egen JG, Huang AY, et al. Extrafollicular activation of lymph node B cells by antigen-bearing dendritic cells. Science 2006; 312:1672-1676.

42 Sohn HW, Tolar P, Jin T, et al. Fluorescence resonance energy transfer in living cells reveals dynamic membrane changes in the initiation of B cell signaling. Proc Natl Acad Sci USA 2006; 103:8143-8148.

43 Sohn HW, Tolar P, Pierce SK. Membrane heterogeneities in the formation of B cell receptor-Lyn kinase microclusters and the immune synapse. J Cell Biol 2008; 182:367-379.

44 Depoil D, Fleire S, Treanor BL, et al. CD19 is essential for $\mathrm{B}$ cell activation by promoting $\mathrm{B}$ cell receptor-antigen microcluster formation in response to membrane-bound ligand. Nat Immunol 2008; 9:63-72.

45 Mattila PK, Feest C, Depoil D, et al. The actin and tetraspanin networks organize receptor nanoclusters to regulate B cell receptor-mediated signaling. Immunity 2013; 38:461-474.

46 Hao S, August A. Actin depolymerization transduces the strength of B-cell receptor stimulation. Mol Biol Cell 2005; 16:2275-2284.

47 Mukherjee S, Zhu J, Zikherman J, et al. Monovalent and multivalent ligation of the B cell receptor exhibit differential dependence upon Syk and Src family kinases. Sci Signal 2013; 6:ra1.

48 Wakabayashi C, Adachi T, Wienands J, et al. A distinct signaling pathway used by the IgG-containing B cell antigen receptor. Science 2002; 298:2392-2395.

49 Horikawa K, Martin SW, Pogue SL, et al. Enhancement and suppression of signaling by the conserved tail of IgG memory-type B cell antigen receptors. J Exp Med 2007; 204:759769.

50 Engels N, Konig LM, Heemann C, et al. Recruitment of the cytoplasmic adaptor Grb2 to surface IgG and IgE provides antigen receptor-intrinsic costimulation to class-switched B cells. Nat Immunol 2009; 10:1018-1025.

51 Sheng M, Sala C. PDZ domains and the organization of supramolecular complexes. Annu Rev Neurosci 2001; 24:1-29.

52 Kim E, Sheng M. PDZ domain proteins of synapses. Nat Rev Neurosci 2004; 5:771-781.

53 Zheng CY, Seabold GK, Horak M, et al. MAGUKs, synaptic development, and synaptic plasticity. Neuroscientist 2011; 17:493-512.

54 Jang IK, Cronshaw DG, Xie LK, et al. Growth-factor receptor-bound protein-2 (Grb2) signaling in B cells controls lymphoid follicle organization and germinal center reaction. Proc Natl Acad Sci USA 2011; 108:7926-7931.

55 Ackermann JA, Radtke D, Maurberger A, et al. Grb2 regulates B-cell maturation, B-cell memory responses and inhibits B-cell Ca2+ signalling. EMBO J 2011; 30:1621-1633.

56 Stork B, Engelke M, Frey J, et al. Grb2 and the non-T cell activation linker NTAL constitute a $\mathrm{Ca}(2+)$-regulating signal circuit in B lymphocytes. Immunity 2004; 21:681-691.

57 Stork B, Neumann K, Goldbeck I, et al. Subcellular localization of Grb2 by the adaptor protein Dok-3 restricts the intensity of Ca2+ signaling in B cells. EMBO J 2007; 26:1140-1149.

58 Hawkins ED, Oliaro J, Kallies A, et al. Regulation of asym- 
metric cell division and polarity by Scribble is not required for humoral immunity. Nat Commun 2013; 4:1801.

59 Shih TA, Meffre E, Roederer M, et al. Role of BCR affinity in $\mathrm{T}$ cell dependent antibody responses in vivo. Nat Immunol 2002; 3:570-575.

60 Kometani K, Nakagawa R, Shinnakasu R, et al. repression of the transcription factor Bach2 contributes to predisposition of IgG1 memory B Cells toward plasma cell differentiation. Immunity 2013; 39:136-147.

61 Benson MJ, Elgueta R, Schpero W, et al. Distinction of the memory B cell response to cognate antigen versus bystander inflammatory signals. J Exp Med 2009; 206:2013-2025.

62 Dogan I, Bertocci B, Vilmont V, et al. Multiple layers of B cell memory with different effector functions. Nat Immunol 2009; 10:1292-1299.

63 Pape KA, Taylor JJ, Maul RW, et al. Different B cell populations mediate early and late memory during an endogenous immune response. Science 2011; 331:1203-1207.

64 Iwakoshi NN, Lee AH, Glimcher LH. The X-box binding protein-1 transcription factor is required for plasma cell differentiation and the unfolded protein response. Immunol Rev 2003; 194:29-38.

65 Klein U, Casola S, Cattoretti G, et al. Transcription factor IRF4 controls plasma cell differentiation and class-switch recombination. Nat Immunol 2006; 7:773-782.

66 Martins G, Calame K. Regulation and functions of Blimp-1 in T and B lymphocytes. Annu Rev Immunol 2008; 26:133-169.

67 Basso K, Dalla-Favera R. Roles of BCL6 in normal and transformed germinal center B cells. Immunol Rev 2012; 247:172183.

68 Cobaleda C, Schebesta A, Delogu A, et al. Pax5: the guardian of B cell identity and function. Nat Immunol 2007; 8:463-470.

69 Igarashi K, Ochiai K, Muto A. Architecture and dynamics of the transcription factor network that regulates B-to-plasma cell differentiation. $J$ Biochem 2007; 141:783-789.

70 Luckey CJ, Bhattacharya D, Goldrath AW, et al. Memory T and memory B cells share a transcriptional program of self-renewal with long-term hematopoietic stem cells. Proc Natl Acad Sci USA 2006; 103:3304-3309.

71 Bhattacharya D, Cheah MT, Franco CB, et al. Transcriptional profiling of antigen-dependent murine $\mathrm{B}$ cell differentiation and memory formation. J Immunol 2007; 179:6808-6819.

72 Tomayko MM, Anderson SM, Brayton CE, et al. Systematic comparison of gene expression between murine memory and naive $\mathrm{B}$ cells demonstrates that memory $\mathrm{B}$ cells have unique signaling capabilities. J Immunol 2008; 181:27-38.

73 Arpin C, Banchereau J, Liu YJ. Memory B cells are biased towards terminal differentiation: a strategy that may prevent repertoire freezing. J Exp Med 1997; 186:931-940.

74 Klein U, Tu Y, Stolovitzky GA, et al. Transcriptional analysis of the B cell germinal center reaction. Proc Natl Acad Sci USA 2003; 100:2639-2644.

75 Tangye SG, Avery DT, Hodgkin PD. A division-linked mechanism for the rapid generation of Ig-secreting cells from human memory B cells. J Immunol 2003; 170:261-269.

76 Good KL, Bryant VL, Tangye SG. Kinetics of human B cell behavior and amplification of proliferative responses following stimulation with IL-21. J Immunol 2006; 177:5236-5247.

77 Bryant VL, Ma CS, Avery DT, et al. Cytokine-mediated regulation of human B cell differentiation into Ig-secreting cells: predominant role of IL-21 produced by CXCR5+ T follicular helper cells. J Immunol 2007; 179:8180-8190.

78 Good KL, Tangye SG. Decreased expression of Kruppel-like factors in memory B cells induces the rapid response typical of secondary antibody responses. Proc Natl Acad Sci USA 2007; 104:13420-13425.

79 Kusumi A, Shirai YM, Koyama-Honda I, et al. Hierarchical organization of the plasma membrane: investigations by single-molecule tracking vs. fluorescence correlation spectroscopy. FEBS Lett 2010; 584:1814-1823.

80 Kusumi A, Nakada C, Ritchie K, et al. Paradigm shift of the plasma membrane concept from the two-dimensional continuum fluid to the partitioned fluid: high-speed single-molecule tracking of membrane molecules. Annu Rev Biophys Biomol Struct 2005; 34:351-378.

81 Kusumi A, Fujiwara TK, Chadda R, et al. Dynamic organizing principles of the plasma membrane that regulate signal transduction: commemorating the fortieth anniversary of Singer and Nicolson's fluid-mosaic model. Annu Rev Cell Dev Biol 2012; 28:215-250.

(c) (i) (3) $\odot$ This work is licensed under the Creative Commons Attribution-NonCommercial-No Derivative Works 3.0 Unported License. To view a copy of this license, visit http:// creativecommons.org/licenses/by-nc-nd/3.0 